Pacific Journal of Mathematics

COLLECTIVELY COMPACT AND SEMI-COMPACT SETS OF
LINEAR OPERATORS IN TOPOLOGICAL VECTOR SPACES 


\title{
COLLECTIVELY COMPACT AND SEMI-COMPACT SETS OF LINEAR OPERATORS IN TOPOLOGICAL VECTOR SPACES
}

\author{
M. V. Deshpande and N. E. Joshi
}

\begin{abstract}
A set of linear operators from one topological vector space to another is said to be collectively compact (resp. semi-compact) if and only if the union of images of a neighbourhood of zero (respectively every bounded set) is relatively compact. In this paper sufficient conditions for a set of operators to be collectively compact or semi-compact are obtained. It is proved that if $T_{n} \rightarrow T$ asymptotically, where $X$ is quasi-complete and $T_{n}$ are $W$-compact then $\left\{T_{n}-T\right\}$ is collectively compact. The final section deals with collectively weakly compact sets. It is proved that in a reflexive locally convex space a family of continuous endomorphisms is collectively weakly compact if and only if
\end{abstract}

$$
\mathscr{K}^{*}=\left\{K^{*}: E_{s}^{*} \longrightarrow E_{w^{*}}^{*}\right\}
$$

is collectively compact.

The concept of collectively compact sets of linear operators on normed linear spaces was introduced by Anselone and Moore [3]. This concept was studied in greater detail by Anselone and Palmer $[1,2]$. Some of the results in these papers were extended to more general spaces in [4]. In this paper some further generalizations are obtained.

2. Let $X$ and $Y$ be topological vector spaces and $\mathscr{L}[X, Y]$, the set of continuous linear operators on $X$ to $Y$. The underlying scalar field will be assumed to be the field of complex numbers, unless otherwise stated.

Definition 2.1. A subset $\mathscr{C} \subset \mathscr{L}[X, Y]$ is said to be collectively compact (respectively, weakly compact, totally bounded) if and only if there exists a neighbourhood $V$ of zero in $X$ such that $\mathscr{K} V=\{K x: K \in \mathscr{L}, x \in V\}$ is relatively compact (respectively weakly compact, totally bounded) in $Y$.

REMARK. It is obvious that $\mathscr{K}$ collectively compact $\Rightarrow \mathscr{K}$ collectively weakly compact. However, if $Y$ is a Montel space, the reverse implication is also true. 
Proposition 2.1. Let $\mathscr{K} \subset \mathscr{L}[X, Y]$ be collectively compact and $Y$, a quasi-complete locally convex space. Then the following statements hold.

(a) The convex hull of $\mathscr{K}$ is collectively compact.

(b) The balanced hull of $\mathscr{K}$ is collectively compact.

(c) The absolutely convex hull of $\mathscr{K}$ is collectively compact.

(d) The closure of $\mathscr{K}$ in the pointwise topology, and therefore in $\mathscr{L}_{b}^{*}[X, Y]$ is collectively compact.

(e) The set $\left\{\sum_{n=1}^{N} \lambda_{n} K_{n}: K_{n} \in \mathscr{K}, \sum_{n=1}^{N}\left|\lambda_{n}\right| \leqq b, b>0, N \leqq \infty\right\}$ is collectively compact, the convergence of the series being in $\mathscr{L}_{b}[X, Y]$.

Proof. (a) Let $\complement \mathscr{K}$ be the convex hull of $\mathscr{K}$. As $\mathscr{K}$ is collectively compact, there exists a neighbourhood $V$ of zero in $X$ such that $\mathscr{C} V$ is relatively compact in $Y$. Now,

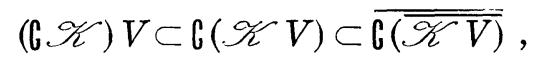

where bar denotes the closure. Since $\overline{\Re V}$ is compact and $Y$ is quasi-complete, $\overline{\complement(\overline{\mathscr{K V})}}$ is compact $[9,20.6(3)]$. It follows that $\mathscr{\complement} \mathscr{K}$ is collectively compact. The proofs of $(b)-(e)$ are similar to those in [1].

Proposition 2.2. Let $X, Y$ and $Z$ be topological vector spaces and $\mathscr{K} \subset \mathscr{L}[X, Y], \mathscr{l} \subset \mathscr{L}[Z, X], \mathscr{N} \subset \mathscr{L}[Y, Z]$ then:

(a) $\mathscr{C}$ collectively compact and $\mathscr{C l}$ equicontinuous $\Rightarrow \mathscr{K}$ KC collectively compact.

(b) $\mathscr{T}$ collectively compact and $\mathscr{N}$ relatively compact in the $\mathscr{C}_{b}[X, Y] \Rightarrow \mathscr{N} \mathscr{C}$ is collectively compact.

Proof. (a) Since $\mathscr{K}$ is collectively compact, there exists a neighbourhood $V$ of zero in $X$ such that $\mathscr{K} V$ is relatively compact in $Y$. Further, by the equicontinuity of $\mathscr{C}$, there exists a neighbourhood $W$ of zero in $Z$ such that $\mathscr{C} W \subset V$. Hence

$$
(\mathscr{K} \mathscr{C}) W \subset \mathscr{\varkappa} V \text {. }
$$

From this the assertion follows.

(b) See [4], Prop. 2.3 (b).

Corollary. If $\mathscr{\mathscr { C }} \subset \mathscr{L}[X, Y]$ is collectively compact and $\mathscr{l l} \subset \mathscr{L}[Z, X]$ is bounded where $Z$ is barreled and $X$ locally convex, then $\mathscr{K} \mathscr{C l}$ is collectively compact.

* $\mathscr{L}[X, Y]$ with the topology of uniform convergence on bounded sets of $X$. 
Proof. For, if $Z$ is barreled and $X$ is locally convex then $\mathscr{C} \subset \mathscr{L}_{b}[Z, X]$ is bounded if and only if it is equicontinuous.

It is proved in [1] that a compact set of compact operators on a Banach space is collectively compact. We shall prove a similar but slightly weaker result for topological vector spaces. For this, we introduce the following definitions.

Definition 2.2. A linear operator $K \in \mathscr{L}[X, Y]$, where $X$ and $Y$ are topological vector spaces, is said to be semi-compact if it maps every bounded subset of $X$ into a relatively compact subset of $Y$.

It is obvious that a compact operator is semi-compact. The converse is also true if $X$ is a quasinormed space.

Definition 2.3. A set of linear operators $\mathscr{K} \subset \mathscr{L}[X, Y]$ is said to be collectively semi-compact, if and only if, for every bounded set $B \subset X, \mathscr{K} B$ is relatively compact in $Y$.

It is clear that a collectively compact set of operators is collectively semi-compact and the propositions proved so far, for collectively compact sets, are also true for collectively semi-compact operators if $X$ is bornological and $Y$ locally convex, because, a semicompact operator is bounded on bounded sets and therefore continuous if the domain space is bornological.

We prove the following

Lemma 2.1. Let $\mathscr{F}$ be an equicontinuous family of operators on a compact set. X into a topolological vector space $Y$. Let $\mathscr{F}$ be compact with respect to the topology of pointwise convergence. Then the set $\mathscr{F}(\mathscr{K})=\{f(K): f \in \mathscr{F}, K \in \mathscr{K}\}$ is compact.

Proof. $\mathscr{F}$ is equicontinuous, therefore, $f(K)$ is jointly continuous, in the sense, that the map $(\mathscr{F} \times \mathscr{T}) \rightarrow Y$ defined by $(f, K) \rightarrow f K$ is continuous relative to the product topology [8, 8.14]. Now $\mathscr{F} \times \mathscr{K}$ is compact, hence $\mathscr{F} \mathscr{K}$, the continuous image of $\mathscr{F} \times \mathscr{K}$ is compact.

The following proposition generalizes the theorem 3.6 in [4].

Proposition 2.3. Let $X, Y$ be locally convex spaces, $X$ bornologic. Let $\mathscr{X}$ be a set of semi-compact operators, compact in $\mathscr{L}_{b}[X, Y]$. Then $\mathscr{K}$ is collectively semi-compact.

Proof. Define a map $f_{x}: \mathscr{L}[X, Y] \rightarrow Y$ by $f_{x}(K)=K x$ for $K \in \mathscr{L}[X, Y]$ and each $x \in B$, a bounded set in $X$. Consider the set 
$\mathscr{F}=\left\{f_{x}: x \in B\right\}$. We prove that $\mathscr{F}$ is equicontinuous. Let $V$ be a neighbourhood of zero in $Y$. Then the set $W=\{K: K B \subset V\}$ is a neighbourhood of zero in $\mathscr{L}_{b}[X, Y]$. Now,

$$
\begin{aligned}
\mathscr{F} W & =\left\{f_{x}(K): f_{x} \in \mathscr{F}, K \in W\right\} \\
& =\{K x: K \in W, x \in B\} \\
& =W(B) \subset V .
\end{aligned}
$$

This proves the equicontinuity of $\mathscr{F}$. Now, the closure $\overline{\mathscr{F}}$ in the pointwise topology is also equicontinuous. Also, $\overline{\mathscr{F}} K \subset \overline{\mathscr{F}} \bar{K}=\overline{K B}$ which is compact by hypothesis on $\mathscr{K}$. Hence $\overline{\mathscr{F}} K$ is relatively compact in $Y$, for each $K \in \mathscr{K}$. From this follows the compactness of $\overline{\mathscr{F}}[8, \S 8$, Problem H]. From Lemma 2.1 we deduce that $\mathscr{F} \mathscr{F} \mathscr{K}$ is compact. But $\mathscr{K} B=\mathscr{F} \mathscr{K} \subset \overline{\mathscr{F}} \mathscr{K}$. Hence $\mathscr{K} B$ is relatively compact. This implies that $\mathscr{K}$ is collectively semi-compact.

COROLlaRY. If $Y$ is complete, then every totally bounded set $\mathscr{C}$ of semi-compact operators in $\mathscr{L}_{b}[X, Y]$ is collectively semicompact.

Proof. In this case $\mathscr{L}_{b}[X, Y]$ is complete. Hence $\overline{\mathscr{K}}$ is compact. By the proposition $\overline{\mathscr{K}}$ is collectively semi-compact. Then so is $\mathscr{K}$.

Proposition 2.4. Suppose $X, Y$ are locally convex spaces. Let $Y$ be reflexive. Then every set $\mathscr{C}$ of semi-compact operators bounded in $\mathscr{L}_{b}[X, Y]$ is collectively weakly semi-compact.

Proof. Since $\mathscr{K}$ is bounded in $\mathscr{L}_{b}[X, Y], \mathscr{K} B$ is bounded for every bounded set $B \subset X$. Since $Y$ is reflexive, every closed bounded set is weakly compact. [10, Th. 36.5]. The conclusion follows.

3. Convergence properties of collectively compact sequences of operators.

Proposition 3.1. Let $X$ and $Y$ be topological vector spaces, $Y$ sequentially complete. Let $T, T_{n} \in \mathscr{L}[X, Y]$ for all $n$. Then:

(a) $T_{n} \rightarrow T$ in $\mathscr{L}_{b}[X, Y]$ if and only if $T_{n} \rightarrow T$ in pointwise topology and $\left\{T_{n}-T\right\}$ is totally bounded in $\mathscr{L}_{b}[X, Y]$.

(b) If, in addition, $X$ is bornologic and $Y$ locally convex, then $T_{n} \rightarrow T$ in $\mathscr{L}_{b}[X, Y]$ and each $T_{n}-T$ semi-compact $\Rightarrow\left\{T_{n}-T\right\}$ is collectively semi-compact. 
Proof. (a) It is evident that $T_{n} \rightarrow T$ in $\mathscr{L}_{b}[X, Y] \quad T_{n} \rightarrow T$ pointwise and $\left\{T_{n}-T\right\}$ is relatively compact, and hence, totally bounded.

For the reverse implication assume that $T_{n}-T \nrightarrow 0$ in $\mathscr{L}_{b}[X, Y]$. This implies that for a given neighbourhood $V$ of zero in $Y$, and bounded set $B$ in $X$, there exists a sequence $n_{i}$ such that $\left(T_{n_{i}}-T\right)(B)$ $\not \subset V$, for each $i=1,2, \cdots$. Since $\left\{T_{n}-T\right\}$ is totally bounded, there exists a Cauchy subsequence $\left\{T_{n_{i j}}-T\right\}$ which must converge in $\mathscr{L}_{b}[X, Y]$ by completeness of $Y$. Because $T_{n}-T \rightarrow 0$ pointwise, it follows that $T_{n_{i_{j}}}-T \rightarrow 0$ in $\mathscr{L}_{b}[X, Y]$. Therefore $\left(T_{n_{i_{j}}}-T\right)(B)$ $\subset V, j>N$, a positive integer. This is a contradiction.

(b) This follows from the fact that a totally bounded set of semi-compact operators is collectively semi-compact if $Y$ is a complete locally convex space and $X$ is bornologic (Cor. Prop. 2.3).

REMARKs. If $T_{n} \rightarrow T$ pointwise and $X$ is of second category, the Banach-Steinhaus theorem implies that the $\left\{T_{n}\right\}$ is equicontinuous, and hence, the pointwise convergence is uniform on the compact sets of $X$. On the other hand, as proved in (a) above, $\left\{T_{n}-T\right\}$ totally bounded and $T_{n} \rightarrow T$ pointwise imply convergence in $\mathscr{L}_{b}[X, Y]$, i.e. uniform convergence on bounded sets. This leads to the following propositions.

Proposition 3.2. Suppose $T_{n} \rightarrow T$ pointwise on $X$, where $X$ is bornologic and of second category. Suppose $\mathscr{K} \subset \mathscr{L}[X, X]$ is collectively semi-compact. Then $\left(T_{n}-T\right) K \rightarrow 0$ in $\mathscr{L}_{b}[X, X]$ uniformly for $K \in \mathscr{K}$.

Proposition 3.3. Let $T_{n} \rightarrow T$ pointwise and $\mathscr{K} \subset \mathscr{L}[X, X]$ be totally bounded in the pointwise topology. Suppose $X$ is complete and of second category. Then $T_{n} K \rightarrow T K$ pointwise uniformly for $K \in \mathscr{K}$.

Proofs. Similar to Propositions 3.1 and 3.2 in [2].

4. Asymptotic convergence and collectively compact sequences of operators.

The concept of convergence of operator sequences in the uniform operator topology in the normed spaces, is generalized in the following manner in [5].

Definition 4.1 A linear operator $K$ on a topological vector space $E$ into itself is said to be the asymptotic limit of a sequence $K_{n}$ of 
linear operators, if and only if, there exists a neighbourhood $V$ of zero in $E$, a sequence $\alpha_{n}$ of scalars $\rightarrow 0$ as $n \rightarrow \infty$ and a bounded set $B \subset E$ such that $\left(K-K_{n}\right) V \subset \alpha_{n} B$, for $n=1,2, \cdots$. This mode of convergence will be denoted by $K_{n} \rightarrow K$, and $K$ will be called the $V$ asymptotic limit of $K_{n}$.

Definition 4.2. A linear operator $K$ on $E$ to itself is said to be $V$-totally bounded if and only if $V$ is a neighbourhood of zero and $K V$ is totally bounded in $E$.

Definition 4.3. If $K$ is the $V$-asymptotic limit of $K_{n}$ and if each $K_{n}$ is $V$-totally bounded, $K$ is said to be asymptotically $V$-totally bounded.

Proposition 4.1. If $K$ is asymptotically $V$-totally bounded, then $K$ is V-totally bounded.

Proof. [5, 4.2-1].

Proposition 4.2. Let $T, T_{n} \in \mathscr{L}[X, X]$ and let $T$ be the $V$ asymptotic limit of $T_{n}$ where each $T_{n}$ is $W$-totally bounded. Then $\left\{T_{n}-T\right\}$ is collectively totally bounded.

Proof. $\quad T_{n} \rightarrow T$ and each $T_{n} W$-totally bounded implies $T$ is $W$ totally bounded (Prop 4.1.). Now, $T_{n} \rightarrow T \Rightarrow$ there exists a sequence $\alpha_{n}$ of scalars $\rightarrow 0$ as $n \rightarrow \infty$, a bounded set $B \subset X$ such that

$$
\left(T_{n}-T\right)(W) \subset \alpha_{n} B \quad \text { for all } n \text {. }
$$

Let $V$ be any neighbourhood of zero. Choose a balanced neighbourhood $V_{1}$ of zero such that $V_{1}+V_{1} \subset V$. Since $B$ is bounded, $B \subset \alpha V_{1}$ for some scalar $\alpha$. Therefore, $\left(T_{n}-T\right)(W) \subset \alpha_{n} \alpha V_{1}$. We can choose $N$ such that $\left|\alpha \alpha_{n}\right|<1$ for $n>N$. Hence $\left(T_{n}-T\right)(W) \subset V_{1}$ for $n>N$. It follows that

$$
\bigcup_{n}\left(T_{n}-T\right)(W) \subset \bigcup_{i=1}^{v}\left(T_{i}-T\right)(W)+V_{1} .
$$

As $\left(T_{i}-T\right)(W)$ is totally bounded for each $i$, so is their finite union. Hence, $\bigcup_{i=1}^{N}\left(T_{i}-T\right)(W) \subset \bigcup_{i=1}^{M}\left(x_{i}+V_{1}\right)$ for some $M$, a positive integer, and $x_{i} \in E$. Hence,

$$
\bigcup_{n}\left(T_{n}-T\right)(W) \subset \bigcup_{i=1}^{M}\left(x_{i}+V\right) .
$$

This proves the proposition. 
Corollary 1. Let $T, T_{n} \in \mathscr{L}[X, X]$ where $X$ is quasi-complete. Suppose $T_{n}$ is $W$-compact i.e. $T_{n}(W)$ is relatively compact for some neighbourhood $W$ of zero in $X$. If $T_{n} \rightarrow T$, then $\left\{T_{n}-T\right\}$ is collectively compact.

Proof. $T_{n} \rightarrow T$ and each $T_{n} W$-compact $\Rightarrow T$ is $W$-compact because $X$ is quasi-complete [5, 4.2-1 Cor. 3]. From the above proposition it follows that $\mathrm{U}_{n}\left(T_{n}-T\right)(W)$ is totally bounded. Hence, the closure $\overline{\mathrm{U}_{n}\left(T_{n}-T\right)(W)}$ is bounded and closed and, therefore, complete by the quasi-completeness of $X$. Thus, $\overline{\mathrm{U}_{n}\left(T_{n}-T\right)(W)}$ is totally bounded and complete, and therefore compact.

Corollary 2. If $T_{n} \rightarrow T$ on a neighbourhood $W$ of zero in $X$, and each $T_{n}$ is $W$-totally bounded, then $\left\{T_{n}-T\right\}$ is collectively compact if $X$ is a Montel space.

Proof. From the Proposition 4.2 it follows that $\left\{T_{n}-T\right\}$ is collectively $W$-totally bounded, and, therefore $W$-collectively compact, as $X$ is a Montel space.

Proposition 4.3. Let $T_{n} \rightarrow T$, where $T_{n}, T \in \mathscr{L}[X, X] . \quad$ If $\mathscr{K} \subset \mathscr{L}[X, X]$ is collectively compact, then $\left(T_{n}-T\right) K \rightarrow 0$ uniformly on $\mathscr{K}$.

Proof. Since $\mathscr{K}$ is collectively compact, there exists a neighbourhood $A$ of zero in $X$ such that $\overline{\mathscr{K} A}$ is compact in $X$, and hence bounded. Now, $T_{n} \rightarrow T \Rightarrow$ there exists a neighbourhood $W$ of zero in $X$, bounded set $B \subset X$, and a sequence $\alpha_{n}$ of scalars $\rightarrow 0$ such that $\left(T_{n}-T\right)(W) \subset \alpha_{n} B$ for all $n$. As $\mathscr{C} A$ is bounded, $\mathscr{C} A \subset r W$ for some scalar $r$. Hence, $\left(T_{n}-T\right)(\mathscr{C} A) \subset\left(T_{n}-T\right)(r W) \subset\left(r \alpha_{n} B\right)$, for all $n$. Since $\alpha_{n}$ and $B$ are independed of $\mathscr{L}$, $\left(T_{n}-T\right) K \rightarrow 0$, uniformly on $\mathscr{K}$.

5. Collectively compact sets in weak topology. In this section we consider the inter-relation between a collectively compact set of operators and its dual family.

Proposition 5.1. Let $E$ be a locally convex topological vector space and $\mathscr{K}$ a family of continuous endomorphisms, uniformly bounded on a neighbourhood $V$ of zero in $E$. Let $\mathscr{K}^{*}$ be the family of dual operators. Then $\mathscr{K}^{*}$ considered as the set of mappings $\left\{K^{*}: E_{s}^{*} \rightarrow E_{w^{*}}^{*}\right\}$ is collectively compact, where $E_{s}^{*}$ is the strong dual and $E_{w^{*}}^{*}$ the $w^{*}$-dual of $E$. 
Proof. By hypothesis, $\mathscr{K} V=B$ is a bounded set in $E$. Consider neighbourhood $W$ of zero in $E_{s}^{*}$ defined by

$$
\begin{aligned}
W & =\left\{f: f \in E_{s}^{*}, \operatorname{Sup}_{y \in B}|\langle y, f\rangle|<1\right\} \\
& =\left\{f: f \in E_{s}^{*}, \operatorname{Sup}_{\substack{x \in V \\
K^{*} \in \mathscr{V}^{* *}}}\left|\left\langle x, K^{*} f\right\rangle\right|<1\right\} .
\end{aligned}
$$

It then follows that

$$
\begin{aligned}
f \in W & \Longrightarrow\left|\left\langle x, K^{*} f\right\rangle\right|<1 \text { for all } x \in V, \text { and } K^{*} \in \mathscr{K}^{*} \\
& \Longrightarrow K^{*} f \in V^{\circ}, \text { the polar of } V \text { in } E \text {, for all } K^{*} \in \mathscr{K}^{*} \\
& \Longrightarrow \mathscr{K}^{*} W \subset V^{\circ} .
\end{aligned}
$$

Now, by the Banach-Alaoglu theorem [8, Th. 17.4], $V^{\circ}$ is $w^{*}-$ compact in $E^{*}$. Hence $\mathscr{K}^{*} W$ is relatively compact in $E_{w^{*}}^{*}$ This completes the proof.

Proposition 5.2. Let $E$ be a semi-reflexive locally convex space and $\mathscr{K}$, a family of continuous endomorphisms on $E$. If $\mathscr{K}$ is uniformly bounded on a neighbourhood $V$ of zero in $E$, then $\mathscr{K}$ considered as a family of operators from $\left(E_{s}^{*}\right)_{s}^{*} \rightarrow\left(E_{s}^{*}\right)_{w^{*}}^{*}$ is collectively compact.

Proof. From Proposition 5.1 it follows that the family $\mathscr{C}^{*}$ of operators from $E_{s}^{*} \rightarrow E_{w^{*}}^{*}$ is collectively compact. Therefore, there exists a neighbourhood $W$ of zero in $E_{s}^{*}$ such that $B=\mathscr{K}^{*} W$ is relatively compact in $E_{w^{*}}^{*}$ and, hence, bounded in $w^{*}$-topology. From semi-reflexivity and from the fact, that a weakly bounded set is also bounded in the initial topology [8, Th. 17.5], it follows that $B$ is bounded in $E$. From Proposition 5.1, it follows that

$$
\mathscr{C}^{* *}=\left\{K^{* *}:\left(E_{s}^{*}\right)_{s}^{*} \longrightarrow\left(E_{s}^{*}\right)_{w^{*}}^{*}\right\}
$$

is collectively compact. Also $\mathscr{K}^{*}=\mathscr{C}^{* *}$ by the continuity of each $K \in \mathscr{K}$. Hence the result.

Corollary. Let $K$ be a continuous linear endomorphism on $E$, a locally convex space. Suppose $K$ is bounded on a neighbourhood of zero in $E$. If $E$ is reflexive, then $K$ is weakly compact.

Proposition 5.3. Let $E$ be a locally convex, reflexive space, and $\mathscr{K}^{2}$ a family of continuous endomorphisms on $E$. Let $\mathscr{K}^{*}$ be the corresponding dual family of endomorphisms on $E^{*}$. Then $\mathscr{K}$ is collectively weakly compact if and only if $\mathscr{K}^{*}$ as the family of 
operators $\left\{K^{*}: E_{s}^{*} \rightarrow E_{w^{*}}^{*}\right\}$ is collectively compact.

Proof. Suppose $\mathfrak{K}^{* *}$ is collectively compact as the family of operators $\left\{K^{*}: E_{s}^{*} \rightarrow E_{w^{*}}^{*}\right\}$. Then there exists a neighbourhood $W$ of zero in $E_{s}^{*}$, such that $\mathscr{K}^{*}(W)$ is relatively $w^{*}$-compact. This implies, since $E$ is reflexive and, therefore barreled, that $\mathscr{K}^{*}(W)$ is equicontinuous, [10, Th. 33.2]. Hence, there exists a neighbourhood $V$ of zero in $E$, such that $\mathscr{K}^{*}(W) \subset V^{\circ}$, the polar of $V$. [10, Prop. 32.7]. Therefore,

$$
\begin{aligned}
& \left|\left\langle K^{*} w, x\right\rangle\right| \leqslant 1, \\
& \quad \text { for all } x \in V, K^{*} \in \mathscr{K}^{*}, w \in W \Longrightarrow \mathscr{K}(V) \subset W^{\circ} .
\end{aligned}
$$

From the reflexivity of $E$ and the Banach-Alaoglu theorem, $\mathscr{K}(V)$ is relatively $w$-compact. This proves that $\mathscr{C}$ is collectively weakly compact.

The converse follows from Proposition 5.1.

COROLlaRY. Let $K$ be a continuous endomorphism on a reflexive locally convex space $E$. Then $K$ is weakly compact if and only if $K^{*}: E_{s}^{*} \rightarrow E_{w^{*}}^{*}$ is compact.

This is a partial generalization of the Theorem 2.13.7 in [7].

\section{REFERENCES}

1. P. M. Anselone and T. W. Palmer, Collectively compact sets of linear operators, Pacific J. Math. 25 (1968), 417-423.

2. - Spectral analysis of collectively compact, strongly convergent operator sequences, Pacific J. Math. 25 (1968), 423-431.

3. P. M. Anselone and R. H. Moore, Approximate solution of integral operator equations, J. Math. Analysis Appl. 9 (1964), 268-277.

4. J. D. DePree and J. A. Higgins, Collectively compact sets of linear operators, Math.

Z., 115 (1970), 366-370.

5. G. F. C. De Bruyn, Asymptotic Properties of Linear Operators, Proc. London Math. Soc. Third Series XVIII (1968), 405-427.

6. R. E. Edwards, Functional Analysis, Holt, Rinehart and Winston, 1965.

7. E. Hille and R. Phillips, Functional Analysis and Semi-groups. Amer. Math. Soc., (1957).

8. J. L. Kelly, I. Namioka et al., Linear Topological Spaces, Van Nostrand, 1968.

9. G. Köthe, Topological Vector Spaces, 1, Springer-Verlag (1969).

10. F. Trèves, Topological Vector Spaces, Academic Press (1967).

Note. After the original draft of this paper was submitted for publication, the authors' attention was drawn to the reference [4] by Prof. Anselone and subsequently, by the referee, for which the authors are thankful. 
11. K. Yosida, Functional Analysis, Springer-Verlag, (1968).

Received June 10, 1971 and in revised form November 30, 1971.

NAGPUR UNIVERSITY, NAGPUR, (MAHARASHTRA) INDIA 


\section{PACIFIC JOURNAL OF MATHEMATICS}

\section{EDITORS}

\author{
H. SAMELSON \\ Stanford University \\ Stanford, California 94305 \\ C. R. HOBBY \\ University of Washington \\ Seattle, Washington 98105
}

\author{
J. DugundJI \\ Department of Mathematics \\ University of Southern California \\ Los Angeles, California 90007
}

\author{
RICHARD ARENS \\ University of California \\ Los Angeles, California 90024
}

\section{ASSOCIATE EDITORS}
E. F. BECKENBACH
B. H. NeumanN
F. WOLF
K. YoSHIDA

\section{SUPPORTING INSTITUTIONS}

\author{
UNIVERSITY OF BRITISH COLUMBIA \\ CALIFORNIA INSTITUTE OF TECHNOLOGY \\ UNIVERSITY OF CALIFORNIA \\ MONTANA STATE UNIVERSITY \\ UNIVERSITY OF NEVADA \\ NEW MEXICO STATE UNIVERSITY \\ OREGON STATE UNIVERSITY \\ UNIVERSITY OF OREGON \\ OSAKA UNIVERSITY
}

\author{
UNIVERSITY OF SOUTHERN CALIFORNIA \\ STANFORD UNIVERSITY \\ UNIVERSITY OF TOKYO \\ UNIVERSITY OF UTAH \\ WASHINGTON STATE UNIVERSITY \\ UNIVERSITY OF WASHINGTON \\ $* * * *$
$*$
AMERICAN MATHEMATICAL SOCIETY
NAVAL WEAPONS CENTER
}

The Supporting Institutions listed above contribute to the cost of publication of this Journal, but they are not owners or publishers and have no responsibility for its content or policies.

Mathematical papers intended for publication in the Pacific Journal of Mathematics should be in typed form or offset-reproduced, (not dittoed), double spaced with large margins. Underline Greek letters in red, German in green, and script in blue. The first paragraph or two must be capable of being used separately as a synopsis of the entire paper. The editorial "we" must not be used in the synopsis, and items of the bibliography should not be cited there unless absolutely necessary, in which case they must be identified by author and Journal, rather than by item number. Manuscripts, in duplicate if possible, may be sent to any one of the four editors. Please classify according to the scheme of Math. Rev. Index to Vol. 39. All other communications to the editors should be addressed to the managing editor, Richard Arens, University of California, Los Angeles, California, 90024.

50 reprints are provided free for each article; additional copies may be obtained at cost in multiples of 50 .

The Pacific Journal of Mathematics is published monthly. Effective with Volume 16 the price per volume (3 numbers) is $\$ 8.00$; single issues, $\$ 3.00$. Special price for current issues to individual faculty members of supporting institutions and to individual members of the American Mathematical Society: $\$ 4.00$ per volume; single issues $\$ 1.50$. Back numbers are available.

Subscriptions, orders for back numbers, and changes of address should be sent to Pacific Journal of Mathematics, 103 Highland Boulevard, Berkeley, California, 94708.

PUBLISHED BY PACIFIC JOURNAL OF MATHEMATICS, A NON-PROFIT CORPORATION

Printed at Kokusai Bunken Insatsusha (International Academic Printing Co., Ltd.), 270, 3-chome Totsuka-cho, Shinjuku-ku, Tokyo 160, Japan. 


\section{Pacific Journal of Mathematics}

\section{Vol. 43, No. 2 \\ April, 1972}

Arne P. Baartz and Gary Glenn Miller, Souslin's conjecture as a problem on the real line....................................... 277

Joseph Barback, On solutions in the regressive isols ............... 283

Barry H. Dayton, Homotopy and algebraic K-theory ................ 297

William Richard Derrick, Weighted convergence in length ............ 307

M. V. Deshpande and N. E. Joshi, Collectively compact and semi-compact sets of linear operators in topological vector spaces ............. 317

Samuel Ebenstein, Some $H^{p}$ spaces which are uncomplemented in $L^{p} \ldots . .327$

David Fremlin, On the completion of locally solid vector lattices ......... 341

Herbert Paul Halpern, Essential central spectrum and range for elements of

a von Neumann algebra............................... 349

G. D. Johnson, Superadditivity intervals and Boas' test ............. 381

Norman Lloyd Johnson, Derivation in infinite planes . . . . . . . . . . . 387

V. M. Klassen, The disappearing closed set property .............. 403

B. Kuttner and B. N. Sahney, On the absolute matrix summability of Fourier series ........................................... 407

George Maxwell, Algebras of normal matrices................... 421

Kelly Denis McKennon, Multipliers of type $(p, p) \ldots \ldots \ldots \ldots \ldots \ldots . . \ldots 29$

James Miller, Sequences of quasi-subordinate functions ............. 437

Leonhard Miller, The Hasse-Witt-matrix of special projective varieties ..... 443

Michael Cannon Mooney, A theorem on bounded analytic functions ...... 457

M. Ann Piech, Differential equations on abstract Wiener space .......... 465

Robert Piziak, Sesquilinear forms in infinite dimensions ............. 475

Muril Lynn Robertson, The equation $y^{\prime}(t)=F(t, y(g(t))) \ldots \ldots \ldots \ldots .483$

Leland Edward Rogers, Continua in which only semi-aposyndetic

subcontinua separate ............................... 493

Linda Preiss Rothschild, Bi-invariant pseudo-local operators on Lie

groups ...................................... 503

Raymond Earl Smithson and L. E. Ward, The fixed point property for

arcwise connected spaces: a correction ...................... 511

Linda Ruth Sons, Zeros of sums of series with Hadamard gaps .......... 515

Arne Stray, Interpolation sets for uniform algebras............... 525

Alessandro Figà-Talamanca and John Frederick Price, Applications of random Fourier series over compact groups to Fourier multipliers .. 